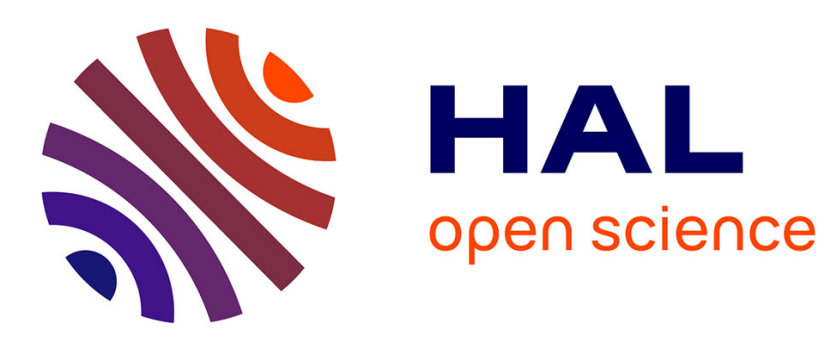

\title{
Transient acoustic wave propagation in non-integer-dimensional rigid porous media.
}

Amine Berbiche, Mohamed Fellah, Zine El Abiddine Fellah, Mustapha

Sadouki, Claude Dépollier

\section{- To cite this version:}

Amine Berbiche, Mohamed Fellah, Zine El Abiddine Fellah, Mustapha Sadouki, Claude Dépollier. Transient acoustic wave propagation in non-integer-dimensional rigid porous media.. Fifth Biot Conference on Poromechanics, Jul 2013, Vienne, Austria. pp.259-266. hal-00868183

\section{HAL Id: hal-00868183 \\ https://hal.science/hal-00868183}

Submitted on 1 Oct 2013

HAL is a multi-disciplinary open access archive for the deposit and dissemination of scientific research documents, whether they are published or not. The documents may come from teaching and research institutions in France or abroad, or from public or private research centers.
L'archive ouverte pluridisciplinaire HAL, est destinée au dépôt et à la diffusion de documents scientifiques de niveau recherche, publiés ou non, émanant des établissements d'enseignement et de recherche français ou étrangers, des laboratoires publics ou privés. 


\title{
Transient acoustic wave propagation in non-integer-dimensional rigid porous
} media.

\author{
A. Berbiche ${ }^{1}$, M. Fellah ${ }^{1}$, Z.E.A. Fellah ${ }^{2}$, M. Sadouki ${ }^{3}$ and C. Depollier ${ }^{4}$ \\ 1 Laboratoire de Physique Théorique, Faculté de Physique, USTHB, BP 32 El \\ Alia, Bab Ezzouar 16111, Algérie, email: amine.berbiche@yahoo.fr, email: mfel- \\ lah@usthb.dz. \\ ${ }^{2}$ LMA, CNRS, UPR 7051, Aix-Marseille Univ, Centrale Marseille, F-13402 Marseille \\ Cedex 20, France, email: Fellah@1ma.cnrs-mrs.fr. \\ ${ }^{3}$ Faculté des Sciences et Technique, Université de Khemis Miliana, Route de Thénia, \\ Khemis Miliana BP 44225, Algérie, email: sad.mus17@yahoo.fr. \\ ${ }^{4}$ LUNAM Universite du Maine. UMR CNRS 6613 LAUM UFR STS Avenue O. Mes- \\ siaen 72085 Le Mans CEDEX 09 France, email: Claude.Depollier@univ-lemans.fr.
}

\begin{abstract}
This study concerns the ultrasonic wave propagation in fractional dimensional rigid porous media. A Biot equivalent fluid model with a non-integer dimensional space is developed using the Stillinger-Palmer-Staravinou formalism. A generalized lossy wave equation is derived and solved analytically in time domain. The coefficients of the propagation equation are constant and depend only on the fractional dimension and the acoustical parameters of the porous material. As in the integer dimension case, the obtained propagation equation contains fractional derivative terms that describe viscous and thermal interactions between the solid and the fluid. The dynamic response of the material is obtained using the Laplace transform method.
\end{abstract}

\section{INTRODUCTION}

Understanding sound wave propagation in porous materials saturated by viscous fluid is important in various applications as architectural acoustics, geophysics, rock mechanics (Allard et al 2009). In these media wave attenuation results from viscous dissipation due to the flow created by the passing wave. The passing wave creates local pressure gradients within the fluid phase, leading to the fluid flow. Because of the complicated structure of most porous materials, the induced fluid flow can take place on various length scales. The real structure of porous media is characterized by a network of interconnected pores forming an extremely irregular geometry. A possible way of describing the complex structure of such media is to use the theory of fractal (Adler 1997) sets with non integral dimension. A fractal is a quantity which displays self-similarity on all scales. In physics, behind this word, we understand object or phenomenon having no characteristic length or having structural details in a hierarchy of 
scales which cannot be described by smooth functions. The object does not need to exhibit exactly the same structure at all scales but the same type of structures must appear on all scales.

Modeling of acoustic propagation in in non-integer-dimensional porous media was initiated by Depollier et al (2008) using the Stillinger (1977), Palmer-Stavrinou (2004) formalism. In this work, we develop a temporal model for the propagation of ultrasonic waves in non-integer-dimensional rigid porous medium. We use the notion of fractional derivatives (Samko et al 1993) to describe the visco-thermal exchange between fluid and structure. An original fractional propagation equation is obtained for the ultrasonic propagation in fractal porous material with rigid structure.

\section{EQUIVALENT FLUID MODEL}

In the acoustics of porous materials, one distinguishes two situations according to whether the frame is moving or not. In the first case, the dynamics of the waves due to the coupling between the solid skeleton and the fluid is well described by the Biot theory (Biot 1956). In air-saturated porous media, the vibrations of the structure can be neglected when the excitation is not very important and the waves can be considered to propagate only in fluid. This case is described by the model of equivalent fluid which is a particular case of the Biot model. Express the Lagrangian density $\mathcal{L}=\mathcal{T}-\mathcal{V}$. The expression of the kinetic energy is given by: $\mathcal{T}=\frac{1}{2} \rho_{f}\left(\frac{\partial u}{\partial t}\right)^{2}$, where $u$ is the fluid displacement, $\rho_{f}$ is the fluid density. The expression of the potential energy $\mathcal{V}$ is given by: $\mathcal{V}=\frac{1}{2} K\left(\frac{\partial u}{\partial x}\right)^{2}$, where $K_{a}$ is the compressibility modulus of the fluid. The Lagrangian density is then written by:

$$
\mathcal{L}=\frac{1}{2} \rho_{f}\left(\frac{\partial u}{\partial t}\right)^{2}-\frac{1}{2} K\left(\frac{\partial u}{\partial x}\right)^{2}
$$

The principle of action for the Lagrangian system depends on the vector field $\varphi(x)$, and spatial and temporal derivatives $\partial_{\mu} \varphi(x)$ is given by the integral

$$
S=\int_{a}^{b} d t L\left(\varphi(x), \partial_{\mu} \varphi(x)\right)
$$

with integration between the initial $a$ and final instant $b$. The action can be written in terms of the Lagrange density by:

$$
L=\int_{\partial \Omega} d^{D} x \mathcal{L}\left(\varphi(x), \partial_{\mu} \varphi(x)\right)
$$

where $D$ is the spatial dimension, and $\partial \Omega$ the border. Where the action

$$
S=\int_{\partial \Omega^{\prime}} d^{D+1} x \mathcal{L}
$$


where $\partial \Omega$ is the boundary for all coordinates. Taking into account the variations and minimizing the action $S$, i.e $\delta S=0$, usual equations of Euler-Lagrange are obtained

$$
\frac{\partial \mathcal{L}}{\partial \varphi}-\partial_{\mu}\left(\frac{\partial \mathcal{L}}{\partial\left(\partial_{\mu} \varphi\right)}\right)=0
$$

where $\mu=x, y, z, t$ and $\varphi=u_{x}, u_{y}, u_{z}$. For propagation along the $x$-axis, We obtain the equation of wave propagation in a lossless medium:

$$
\frac{\partial^{2} u(x, t)}{\partial x^{2}}-\left(\frac{\rho_{f}}{K_{a}}\right) \frac{\partial^{2} u(x, t)}{\partial t^{2}}=0
$$

Within the framework of the acoustic propagation in a porous rigid structure, the density and compressibility of the fluid are "renormalized" by the fluid-structure interactions: $\rho_{f} \rightarrow \rho_{f} \alpha(\omega)$ and $K_{a} \rightarrow K_{a} / \beta(\omega)$ giving the following Helmholtz equation in lossy porous material with rigid structure:

$$
\frac{\partial^{2} u(x, t)}{\partial x^{2}}+\omega^{2}\left(\frac{\rho_{f} \alpha(\omega) \beta(\omega)}{K_{a}}\right) u(x, t)=0 .
$$

A prediction of the acoustic comportment of the porous material requires the determination of the dynamic tortuosity $\alpha(\omega)$ and dynamic compressibility $\beta(\omega)$. Theses functions depends to the physical characteristic of the fluid in the pore space of the medium and are independent of the dynamic characteristics of the structure. The functions $\alpha(\omega)$ and $\beta(\omega)$ express the viscous and thermal exchanges between the fluid and the structure which are responsible of the sound damping in acoustic materials. These exchanges are due on the one hand to the fluid-structure relative motion and on the other hand to the fluid compressions-dilatations produced by the wave motion. The parts of the fluid affected by these exchanges can be estimated by the ratio of a microscopic characteristic length of the media, as for example the sizes of the pores, to the viscous and thermal skin depth thickness $\delta=\left(2 \eta / \omega \rho_{f}\right)^{1 / 2}$ and $\delta^{\prime}=\left(2 \eta / \omega \rho_{f} P_{r}\right)^{1 / 2}$. For the viscous effects this domain corresponds to the region of the fluid in which the velocity distribution is perturbed by the frictional forces at the interface between the viscous fluid and the motionless structure. For the thermal effects, it corresponds to the fluid volume affected by the heat exchange between the two phases of the porous medium.

\section{FRACTIONAL MODEL}

When the wave frequency is high, the skin depth is very narrow and the viscous effects are concentrated in a small volume near the frame $\delta / r \ll 1$; then the viscous effects in the fluid can be neglected: the fluid behaves almost like a perfect fluid (without viscosity). In the same way, the compression/dilatation cycle is a much faster process than the heat transfer between the air and the structure and it is a well-founded 
approximation to consider that the compression is adiabatic. In The high frequency approximations (Johnson et al 1987) of the response factors $\alpha(\omega)$ and $\beta(\omega)$ are :

$$
\begin{aligned}
& \alpha(\omega)=\alpha_{\infty}\left(1-\frac{2}{\Lambda}\left(\frac{\eta}{j \omega \rho_{f}}\right)^{1 / 2}\right), \quad \omega \longrightarrow \infty . \\
& \beta(\omega)=1-\frac{2(\gamma-1)}{\Lambda^{\prime}}\left(\frac{\eta}{P_{r} \rho_{f}}\right)^{1 / 2}\left(\frac{1}{j \omega}\right)^{1 / 2}, \quad \omega \longrightarrow \infty,
\end{aligned}
$$

From these two equations, we note that the frequency dependence of $\alpha(\omega)$ and $\beta(\omega)$ is the high frequency range, is in square root of the frequency It is interesting to note that the temporal equivalent of the square root is the fractional derivative. Write the equations in the time domain is equivalent to taking the inverse Fourier transform of (8) and (9). The temporal equivalent of $j \omega$ is $\partial / \partial t$, while the temporal equivalent of $\sqrt{j \omega}$ is a fractional derivative of order $1 / 2$. The definition of fractional derivative of order $\nu$, where $0 \leq \nu<1$ is given by Samko et al (1993):

$$
D^{\nu}[x(t)]=\frac{1}{\Gamma(-\nu)} \int_{0}^{t}(t-u)^{-\nu-1} x(u) d u,
$$

where $\Gamma(x)$ is the Eulerian function of the second kind. From the definition (10), the expressions of response factors $\alpha(\omega)$ and $\beta(\omega)$ are then given in the time domain by (Fellah and Depollier 2000):

$$
\begin{aligned}
& \alpha(\omega) \stackrel{t}{\longrightarrow} \tilde{\alpha}(t)=\alpha_{\infty}\left(\delta(t)+\frac{2}{\Lambda}\left(\frac{\eta}{\pi \rho_{f}}\right)^{1 / 2} t^{-1 / 2}\right), \\
& \beta(\omega) \stackrel{t}{\longrightarrow} \tilde{\beta}(t)=\delta(t)+\frac{2(\gamma-1)}{\Lambda^{\prime}}\left(\frac{\eta}{\pi \operatorname{Pr} \rho_{f}}\right)^{1 / 2} t^{-1 / 2},
\end{aligned}
$$

where $\delta(t)$ is the Dirac distribution and the operators. In this model, the time convolution of $t^{-1 / 2}$ with a function, is interpreted as an operator of fractional derivative. Using Eqs. (11) and (12), the Helmholtz equation (7) becomes in time domain a Fractional wave equation, and is of the form:

$$
\frac{\partial^{2} p(x, t)}{\partial x^{2}}-A \frac{\partial^{2} p(x, t)}{\partial t^{2}}-B \frac{\partial^{3 / 2} p(x, t)}{\partial t^{3 / 2}}-C \frac{\partial p(x, t)}{\partial t}=0,
$$

where the coefficients $A, B$ and $C$ are constants respectively given by ;

$$
A=\frac{\rho_{f} \alpha_{\infty}}{K_{a}}, \quad B=\frac{2 \alpha_{\infty} \sqrt{\rho_{f} \eta}}{K_{a}}\left(\frac{1}{\Lambda}+\frac{\gamma-1}{\sqrt{\operatorname{Pr}} \Lambda^{\prime}}\right) \quad C=\frac{4 \alpha_{\infty}(\gamma-1) \eta}{K_{a} \Lambda \Lambda^{\prime} \sqrt{\operatorname{Pr}}} .
$$

The fractional propagation equation (13) is valid for the case of a porous media with integer-dimension in space We will discuss in the next chapter the case of a medium with non-integer dimension space. Solving this equation for propagation (Fellah et al, $2003,2005)$ in the time domain was crucial for the characterization of porous materials. 


\section{INTEGRATION IN SPACES WITH NON INTEGER DIMENSION}

Stillinger (1977) developed a formalism for writing the Laplace operator in spaces having a fractional dimension D. This dimension is defined from an integral calculus. Let us consider the integration of a radially symmetric function $f$ in a $D$ dimension space:

$$
\int d x_{0} f\left(r\left(x_{0}, x_{1}\right)\right)=\int_{0}^{\infty} d r v_{1}(r) f(r)
$$

where $r\left(x_{0}, x_{1}\right)$ is the distance between points $x_{0}$ and $x_{1}$. Here:

$$
v_{1}(r)=\sigma(D) r^{D-1} \quad \text { and } \quad \sigma(D)=\frac{2 \pi^{D / 2}}{\Gamma(D / 2)},
$$

$D$ is an integer, $\sigma(D)$ agrees with the volume of the unit sphere in Euclidean spaces. This justifies the generalization of fractional dimension to any value of $D$. Using this formalism, Stillinger shows that the Laplace operator in a $D$-dimensional space is

$$
\nabla^{2} f(r)=\frac{\partial^{2} f(r)}{\partial r^{2}}+\left(\frac{D-1}{r}\right) \frac{\partial f(r)}{\partial r}
$$

For a non integer $D$-dimensional space, the Stillinger's formalism leads to a Laplace operator for which the non integer dimension is located in only in one direction. For example, in a space where only the dimension of the $p$ coordinate is integer, the Laplacian becomes

$$
\nabla^{2} f(p, l)=\left[\frac{\partial^{2}}{\partial p^{2}}+\frac{\partial^{2}}{\partial l^{2}}+\frac{D-2}{l} \frac{\partial}{\partial l}\right] f(p, l)
$$

\section{ULTRASONIC WAVE IN FRACTIONAL DIMENSIONAL SPACE}

The Stillinger's formalism of noninteger dimensional spaces has been generalized to $n$ orthogonal coordinates by C. Palmer and P.N. Stavrinou (2004). Using the variational principle, the authors derive the Euler Lagrange equations of a field theory in such spaces which follow from the stationarity property of the action integral with the respect to variations of the fields and their derivatives. So, if the action is defined by

$$
S=\int \mathcal{L} d v d t
$$

where $\mathcal{L}=\mathcal{L}\left(\varphi_{k}, \partial_{\mu} \varphi_{k}\right)$ is the Lagrangian density corresponding to a definite point of the space-time, the Euler-Lagrange equations are:

$$
\frac{\partial \mathcal{L}\left(\varphi_{i}, \partial_{\mu} \varphi_{i}\right)}{\partial \varphi_{i}}-\partial_{\mu} \frac{\partial \mathcal{L}\left(\varphi_{i}, \partial_{\mu} \varphi_{i}\right)}{\partial\left(\partial_{\mu} \varphi_{i}\right)}-\left(d_{\mu \nu}-\delta_{\mu \nu}\right)\left(x^{(-1)}\right)^{\nu} \frac{\partial \mathcal{L}\left(\varphi_{i}, \partial_{\mu} \varphi_{i}\right)}{\partial\left(\partial_{\mu} \varphi_{i}\right)}=0
$$


Here, $i=1,2, \ldots, n$ is the number of degrees freedom (i.e. scalar fields), the index $\mu$ runs from 1 to $4, x^{\nu}=\left(x^{1}, x^{2}, x^{3}, x^{4}=t\right)$ and $\partial_{\mu} \varphi_{i}=\partial \varphi_{i} / \partial x^{\mu} . d$ is a diagonal matrix, the elements of which are the time and spatial dimension $d=\operatorname{diag}\left(1, d_{11}, d_{22}, \ldots, d_{n n}\right)$, with $D=\operatorname{Tr}(d)-1$ and $\delta$ is the diagonal unit matrix. The third term of the left hand side of (19) is the additional term due to the fractional dimension. In an Euclidean space where $d_{\mu \nu}=\delta_{\mu \nu}$ this term vanishes.

The Euler-Lagrange fractional dimension are constructed so as equivalent to those belonging to the entire dimensions, except the introduction of the Lagrangian derivatives multiplied by a constant fraction.

The parameters $\left(d_{\mu \nu}-\delta_{\mu \nu}\right)\left(x^{(-1)}\right)^{\nu} \frac{\partial \mathcal{L}\left(\varphi_{i}, \partial_{\mu} \varphi_{i}\right)}{\partial\left(\partial_{\mu} \varphi_{i}\right)}$ introduce the notions of material anisotropy. In our case, we take $\delta_{\mu \nu}=0$, when $\mu \neq \nu$ and $\delta_{\mu \nu}=1$, when $\mu=\nu$. Moreover, we impose $d_{\mu \nu}=0$, when $\mu \neq \nu$, at least $d_{y y}=d_{z z}=d_{t t}=1$. The fractional dimension is therefore only fixed by the constant $d_{x x}$. However, $d_{x x}+d_{y y}+d_{z z}=D$ and $D \leq 3$, therefore $0 \leq d_{x x} \leq 1$ and $d_{x x}$ is real. To simplify writing, we will take $d_{x x}=d$.

Using the expression of the Lagrangian density(1), the temporal expressions of the tortuosity and compressibility $(8,9)$, we obtain the following fractional propagation equation in fractal porous material, at high frequency range.

$$
\frac{\partial^{2} p(x, t)}{\partial x^{2}}-A \frac{\partial^{2} p(x, t)}{\partial t^{2}}-B \frac{\partial^{3 / 2} p(x, t)}{\partial t^{3 / 2}}-C \frac{\partial p(x, t)}{\partial t}+\left(\frac{d-1}{x}\right) \frac{\partial p(x, t)}{\partial x}=0,
$$

where $d$ is the fractal dimension of the porous material. Eq. (20) is the generalized propagation equation for lossy non-integer-dimensional fractal porous material. This equation is very important for treating the direct and inverse scattering problems in inhomogeneous porous materials in time domain. It is easy to find the special case of integer-dimensional rigid porous media, i.e. when $d=0$, Eq. (20) is reduced to the propagation equation in integer-dimensional material (Eq. 13).

Let us study the sensitivity of the fractal dimension of the propagation equation. Fig. 1 shows the incident signal on a plastic foam sample having the following characteristics: thickness $5 \mathrm{~cm}$, tortuosity $\alpha_{\infty}=1.04$, viscous characteristic length $\Lambda=200 \mu m$, thermal characteristic length $\Lambda^{\prime}=600 \mu m$. Fig. 2 shows a comparison between different signals propagating through the fractal porous medium, for different values of the fractal dimension $d$. From theses figures, we can see that by increasing the value of $d$, the amplitude of the signal decreases, and thus the ultrasonic attenuation of the material increases. This result is very important for solving direct and inverse problems for ultrasonic characterization of porous fractal. We will try, in future work, to solve the inverse problem for measuring the fractal dimension of porous materials using experimental ultrasonic data. 


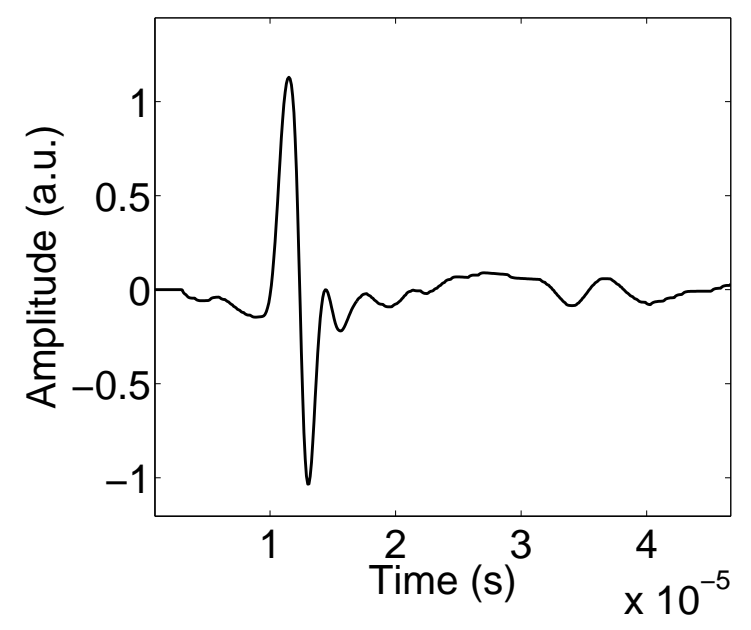

Figure 1. Incident signal.

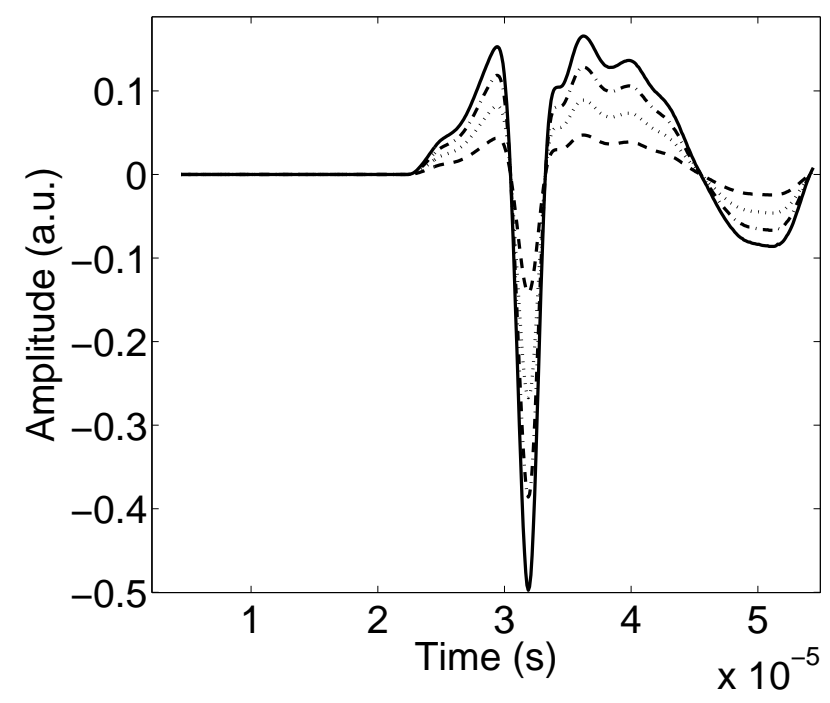

Figure 2. Comparison of signals propagating through the fractal porous medium, for $d=0.2$ (solid line), $d=0.4$ (dashdot line), $d=0.6$ (dotted line) and $d=0.8$ (dashed line)

\section{CONCLUSION}

An original fractional propagation equation is established for the ultrasonic propagation in non-integer-dimensional rigid porous media. The coefficients of this equations depends on the acoustic properties of the porous material and to the fractal 
dimension of the medium. By increasing the fractal dimension of the material, the ultrasonic attenuation of the material increases. The direct problem is solved, we hope, in future solve the inverse problem for the ultrasonic characterization of fractal porous materials.

\section{REFERENCES}

Allard J. F. and Atalla N., (2009) Chapter 5, Propagation of Sound in Porous Media: Modeling Sound Absorbing Materials J. Willey and Sons Ltd, Chichester, West Sussex, PO19 8SQ, United Kingdom.

Adler P.M. (1997), 'Transport processes in Fractals", Int. J. Multiphase Flow, 11, 575605.

Depollier C and Legland J.B (2008), "Wave equation in non-integer-dimensional porous media", Proceeding Acoustics08 Paris 2535-2538.

Stillinger F.H. (1977), "Axiomatic basis for spaces with noninteger dimension", $J$. Math. Phys, 18, 1224-1234.

Palmer C and Stavrinou P.N (2004), "Equations of motion in a non-integer-dimensional space", J. Phjys. A: Math. Gen 37, 6987-7003.

Samko S. G., Kilbas A.A. and Marichev O.I. (1993), "Fractional Integrals and Derivatives : Theory and Applications", Gordon and Breach Science Publishers, Amsterdam 93-112.

Biot M.A (1956), "The theory of propagation of elastic waves in fluid-saturated porous solid. I. Higher frequency range", J. Acoust. Soc. Am. 28, 179-191.

Johnson D.L., Koplik J. and Dashen R. (1987), "Theory of dynamic permeability and tortuosity in fluid-saturated porous media", J. Fluid.Mech. 176, 379-402.

Fellah Z.E.A and Depollier C. (2000), 'Transient acoustic wave propagation in rigid porous media: A time-domain approach", J. Acoust. Soc. Am. 107, 683-688.

Fellah Z.E.A., Fellah M., Lauriks W., Depollier C., Chapelon J-Y., Angel Y.C., (2003) "Solution in time domain of ultrasonic propagation equation in a porous material", Wave Motion 38, 151-163.

Fellah Z.E.A., Depollier C., Fellah M., Lauriks W., Chapelon J-Y. (2005), 'Influence of dynamic tortuosity and compressibility on the propagation of transient waves in porous media", Wave Motion 41, 145-161. 